\title{
Endoscopic Ultrasound-guided Biopsy of Liver Tumors
}

\author{
VLAD ANDREI ICHIM ${ }^{1}$, ROMEO IOAN CHIRA ${ }^{1}$, GEORGIANA ANCA NAGY ${ }^{1}$, \\ ALEXANDRA CHIRA $^{2}$ and PETRU ADRIAN MIRCEA ${ }^{1}$ \\ ${ }^{1} 1^{\text {st }}$ Medical Clinic, Department of Internal Medicine, \\ "Iuliu Hatieganu" University of Medicine and Pharmacy, Cluj-Napoca, Romania; \\ ${ }^{2} 2^{\text {nd }}$ Medical Clinic, Department of Internal Medicine, \\ "Iuliu Hatieganu” University of Medicine and Pharmacy, Cluj-Napoca, Romania
}

\begin{abstract}
Background/Aim: Endoscopic ultrasound (EUS)guided liver tumor biopsy has some advantages over the percutaneous and surgical route and, in many cases, should be preferred. The aim of this study was to evaluate the role of EUS-fine needle aspiration (FNA) in the diagnosis of liver tumors with an emphasis on its diagnostic accuracy and histological quality of the acquired specimen. Patients and Methods: We followed 30 consecutive patients who underwent liver tumor biopsy using EUS guidance. Tissue was acquired using a 22-gauge FNA needle. Results: In 97\% of patients, the results of EUS-FNA were adequate for diagnosis. In one case, the pathologist recommended a repeat biopsy. The acquired specimen was a core fragment in $81 \%$ of cases while in $19 \%$ of cases the specimen was fragmented and subsequently used as a cell block. No complications were reported. Conclusion: EUS-FNA is characterized by a high success rate on the acquisition of good-quality tissue specimens, a low rate of complications, and decreased patient discomfort. This procedure should be especially considered in the case of liver lesions that are inaccessible via the percutaneous route or when concurrent biopsies are required for accurate diagnosis.
\end{abstract}

With all the improvements in imaging techniques, there still remains an important role for liver biopsy in the management of liver tumors. In recent years, an increasing number of reports have been published describing the use of endoscopic

This article is freely accessible online.

Correspondence to: Romeo Ioan Chira, 1 $1^{\text {st }}$ Medical Clinic, Department of Internal Medicine, "Iuliu Hatieganu" University of Medicine and Pharmacy of Cluj-Napoca, 3-5 Clinicilor street, Cluj Napoca 40000, Romania. Tel: +40 751130280, Fax: +40 264592771, e-mail: romeochira@yahoo.com

Key Words: Endoscopic ultrasound-fine needle aspiration (EUSFNA), liver biopsy, focal liver lesions ultrasound - fine needle aspiration (EUS-FNA), as a complementary diagnostic tool for focal liver lesions that cannot be properly targeted by other means.

The European Society of Gastrointestinal Endoscopy (ESGE) suggests performing EUS-guided sampling if the histopathological result is likely to affect the case management and $\mathrm{i}$ ) the lesion is poorly accessible via percutaneous ultrasound or ii) a sample obtained via the percutaneous route repeatedly produces an inconclusive result (1).

Percutaneous ultrasound-guided liver tumor biopsy is recognized as a highly established method, however (2), in certain instances, where the visualization or access to the tumor is limited, it is technically impossible to perform. The advantages of EUS guidance include: i) better accessibility in cases where the percutaneous approach is difficult, ii) fewer complications, iii) the possibility of concurrent biopsies from other sites (pancreas, adrenal, lymph nodes), and iv) higher patient comfort due to the use of sedation.

Despite the benefits EUS-FNA, there are many factors that may affect the quality of the biopsy specimen acquired. Cytology is considered the standard for EUS-FNA-based tissue diagnosis. Although it can detect cellular findings that suggest malignancy, it has limited value in analyzing entities that call for histological and immunophenotypic characterization, which often requires larger, core fragments (3). Nonetheless, with the advancement in the design of needles purposed for EUS-guided biopsies, we can now obtain core fragments with retained architecture and less tissue fragmentation, even when thinner than usual needles are used $(4,5)$.

The aim of this study was to evaluate the role of EUSFNA in the diagnosis of liver tumors with an emphasis on the diagnostic accuracy and histological quality of specimens acquired using a $22 \mathrm{~g}$ FNA needle.

\section{Patients and Methods}

Thirty patients who underwent liver tumor biopsy assisted by EUS guidance were followed between March 2020 and March 2021. The 
Table I. Patient demographics and characteristics of liver lesions.

\begin{tabular}{lc}
\hline Characteristics & Number \\
\hline Gender (male/female) & $17 / 13(57 \% / 43 \%)$ \\
Average age & 64.3 years \\
Tumor location (left lob/right lobe) & $23 / 7(77 \% / 33 \%)$ \\
Tumor number (single/multiple) & $9 / 21(30 \% / 70 \%)$ \\
Tumor diameter (average) & $41 \mathrm{~mm}($ range $=8-95 \mathrm{~mm})$ \\
\hline
\end{tabular}

indication for the procedure was liver tumors that were poorly accessible by percutaneous imaging and the histopathological result was likely to affect the case management, as recommended by the ESGE guidelines. We included patients $>18$ years old, regardless of liver function or underlying cirrhosis. Patients with large volume ascites underwent a paracentesis prior to the procedure. The exclusion criteria were: i) coagulopathy (international normalized ratio $>1.5$ ) or platelet count $<80,000 / \mathrm{mm}^{3}$ and ii) contraindication for deep sedation. The risk for performing an EUS-FNA procedure is based on the risk of hemorrhage. According to the ESGE guidelines (6), i) oral anticoagulant treatment needs to be temporarily substituted with low-molecular weight heparin, ii) $\mathrm{P} 2 \mathrm{Y} 12$ receptor antagonists, such as clopidogrel, have to be stopped five days before the procedure after liaising with a cardiologist in patients with a high thrombotic risk, and iii) aspirin should be continued.

Written informed consent was given by all patients at admission and before the procedure. The study was approved by the "Iuliu Hatieganu" University of Medicine \& Pharmacy Ethics Commission (no.390).

For the procedure, all patients were deeply sedated using propofol with the assistance of an anesthesiologist. EUS was performed using a linear array echoendoscope (Olympus GFUCT180 with ultrasound processor EU-ME 2, Olympus Corporation, Tokyo, Japan). Tissue acquisition was performed with a 22-gauge needle (Micro-tech Endoscopy - Premium(c) EUS-FNA, Micro-tech, Nanjing, PR China) with the following characteristics: i) Cobalt-Cromium alloy needle, ii) maximum needle length $=8 \mathrm{~cm}$, iii) channel size $>2.8 \mathrm{~mm}$, iv) maximum insertion portion $=1.8 \mathrm{~mm}$, v) working length $=137.5-141.5 \mathrm{~cm}$.

We identified the target liver lesions in B-mode and further characterized them with real-time Doppler and strain elastography. Afterwards, we performed FNA from the stomach (for lesions located in the left lobe of the liver) or the duodenum (for lesions located in the right lobe). The needle was inserted into the target lesion under EUS guidance. Once the lesion was punctured, the stylet was detached. The specimen was obtained by moving the needle back and forth more than 10 times inside the lesion while applying intermittent negative pressure using a $20 \mathrm{ml}$ syringe. After this step, suction was released by closing the syringe lock, and the needle was subsequently removed. Aspirated specimens were displaced by reinserting the stylet. The fragment obtained was immersed in formalin and inspected in order to evaluate if additional passes were needed. An adequate tissue specimen was defined as having sample material sufficient to establish a diagnosis at the histological level. In some cases, fragmented material left over in the needle was also laid out onto glass slides for cytology smears.

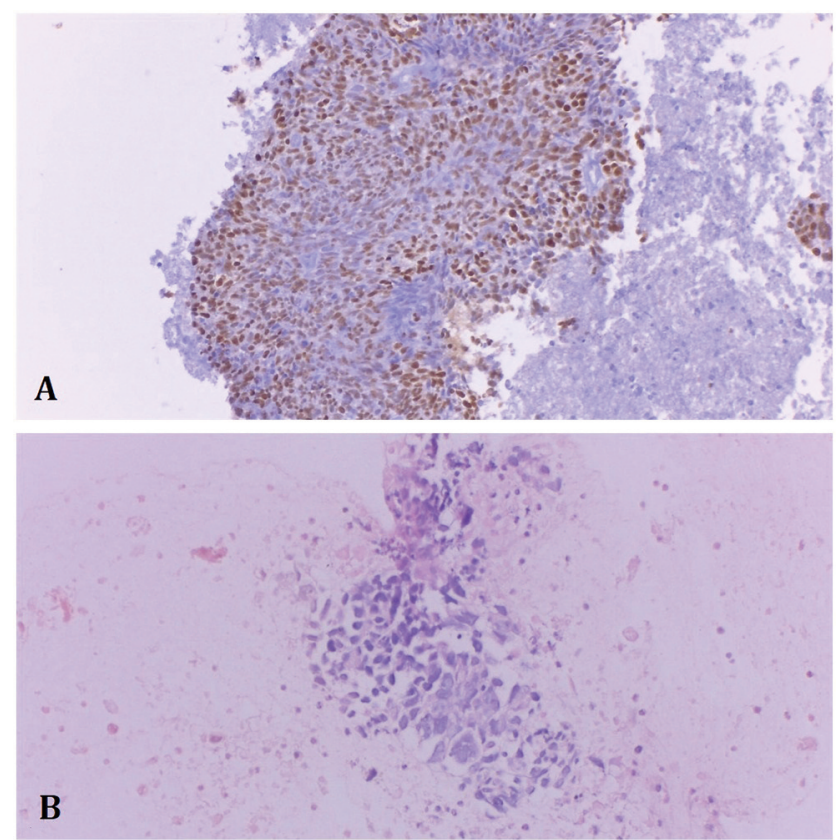

Figure 1. EUS-FNA from malignant liver tumors. A) Poorly differentiated squamous cell carcinoma stained with a p63 antibody (brown color). B) Malignant epithelial cells (Hematoxylin-eosin stain). EUS-FNA: Endoscopic ultrasound-fine needle aspiration. Magnification: $\times 40(A) \times 20(B)$

All patients were admitted for the procedure and followed for complications by clinical examination for $24 \mathrm{~h}$. If any new symptoms occurred, abdominal ultrasound and standard bloodwork was performed.

Histology and immunohistochemistry. Initially, formalin-fixed and paraffin-embedded specimens were analyzed using hematoxylineosin staining by the same experienced pathologist. If haematoxilineosin staining was not sufficient for establishing the diagnosis, immunohistochemistry was performed, using a large array of commercial ready-to-use antibodies (e.g., p63 - clone 4A4, Master Diagnostica, Granada, Spain), depending on the particularities of the tumor and on the recommendations of the pathologist. Immunohistochemical staining was performed using an automated system (MD Steiner Tunic, Bucharest, Romania) according to manufacturer's specifications. Fragmented specimens were prepared as a cell block. Material left over in the needle was used for smears.

Statistical analyses. For our analyses, we performed a Chi-squared test to investigate if there was a statistically significant difference between fragment adequacy obtained via EUS-FNA of the left lobe or right lobe and in relation to tumor size using Microsoft Excel 2017.

\section{Results}

We enrolled 30 patients with liver tumors that were difficult to biopsy percutaneously either due to their small size or 


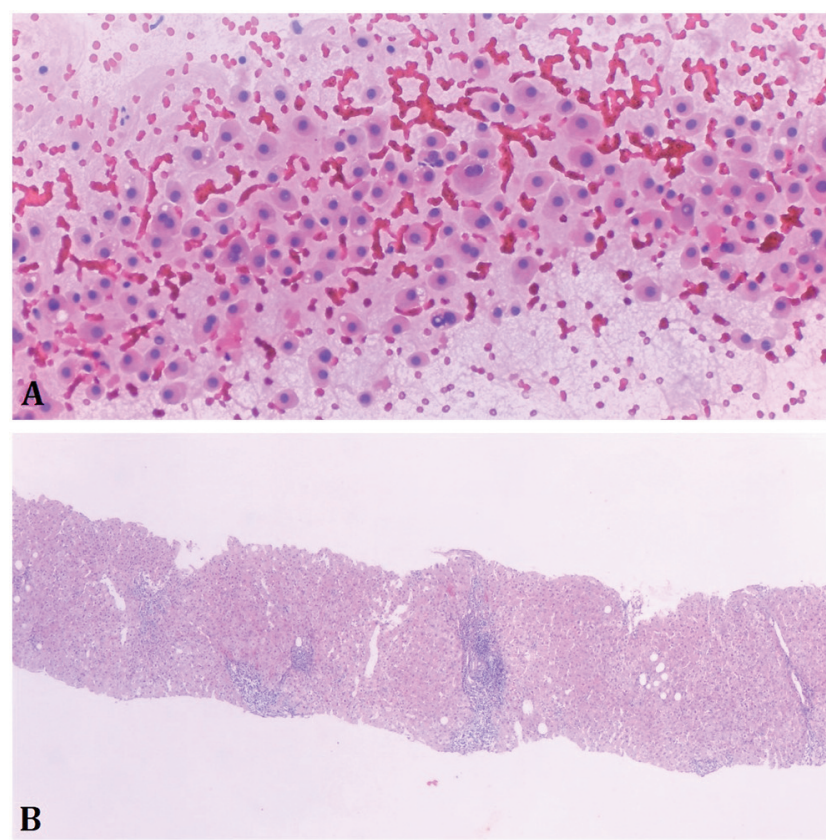

Figure 2. The single EUS-FNA fragment that was considered inadequate for diagnosis - Cell block. A) Hepatocytes without atypia. (Hematoxylin-eosin stain) B) Percutaneous liver biopsy from the same tumor - Chronic hepatitis, portal and periportal lymphocytes (Hematoxylin-eosin stain). EUS-FNA: Endoscopic ultrasound- fine needle aspiration. Magnification: $\times 20($ A) $\times 10(B)$.

long distance from the puncture site. Patient demographics and characteristics of the liver lesions are presented in Table I.

In $29 / 30$ (97\%) of patients, the results of EUS-FNA were adequate for diagnosis, with $27 / 30(90 \%)$ being malignant (Figure 1) and 2/30 (7\%) being benign. In one case, the acquired fragment was considered inadequate by the pathologist and a repeat biopsy was recommended. The same lesion was also biopsied percutaneously, which also did not reveal any signs of malignancy either (Figure 2).

The acquired specimen was a core fragment in $81 \%$ of cases, while in $19 \%$ of cases the specimen was fragmented (Figure 3) and subsequently used as a cell block. The diagnoses for the 30 liver biopsies are documented in Table II.

In patients with metastatic liver lesions, we also biopsied the primitive tumor (12/16 cases). The results from the biopsies of the primary tumor and the liver metastasis were consistent. In some instances, EUS uncovered extrahepatic lesions with signs of malignancy. Those lesions were also biopsied using EUS guidance, using the same procedure as the liver tumor biopsy (Table III).

Cytological evaluation was performed in 3 cases. In 2/3 cases cytology did not reveal malignant cells, while in 1 case it revealed malignant cells, without, though, determining the precise diagnosis (Figure 4).
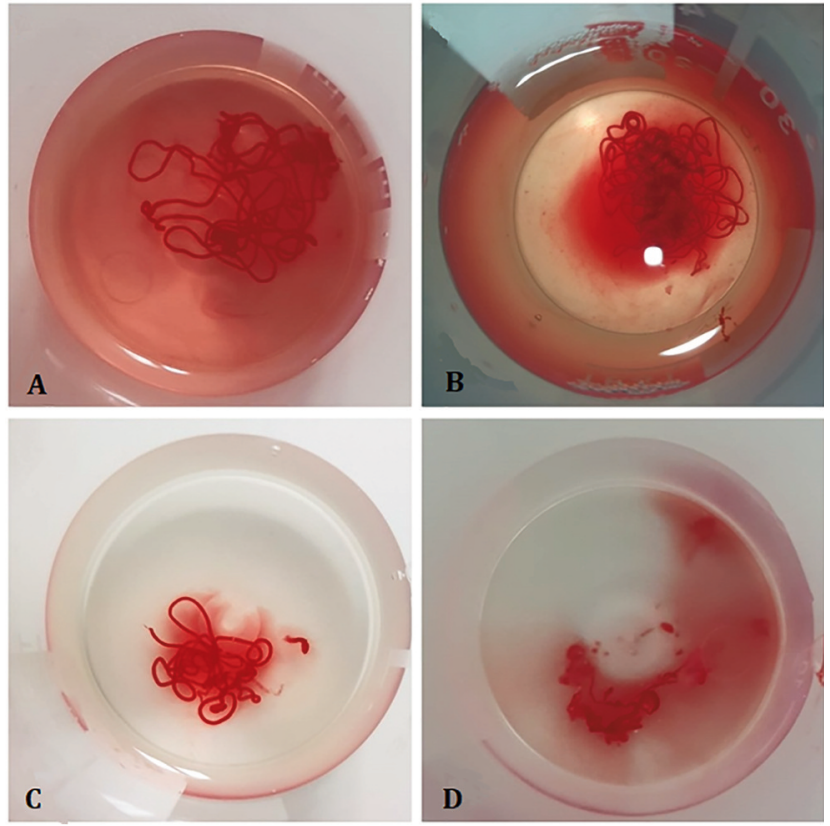

Figure 3. Tissue specimens obtained through EUS-FNA from liver tumors using a $22 G$ needle. A-C) Core specimens and D) fragmented specimen that was used for cell block. EUS-FNA: Endoscopic ultrasound- fine needle aspiration.

Table II. Diagnosis of hepatic tumors obtained through EUS-FNB.

\begin{tabular}{lc}
\hline Tumor types & Number $(\%)$ \\
\hline Metastatic liver lesions & $16(53 \%)$ \\
Pancreatic adenocarcinoma & $3(10 \%)$ \\
Pulmonary adenocarcinoma & $2(7 \%)$ \\
Mammary carcinoma & $1(3 \%)$ \\
Neuroendocrine tumor & $1(3 \%)$ \\
Colon adenocarcinoma & $3(10 \%)$ \\
Squamous cell carcinoma of the cervix & $1(3 \%)$ \\
Lymphoma & $2(7 \%)$ \\
Melanoma & $2(7 \%)$ \\
Anal squamous cell carcinoma & $1(3 \%)$ \\
Primitive liver malignancies & $11(37 \%)$ \\
Cholangiocarcinoma & $6(20 \%)$ \\
Hepatocellular carcinoma & $5(17 \%)$ \\
Benign & $2(7 \%)$ \\
Non-diagnostic & $1(3 \%)$ \\
Total & $30(100 \%)$ \\
\hline
\end{tabular}

The only biopsy fragment that was considered inadequate was obtained from the right lobe. Using Chi-squared contingency test, we determined there is no statistically significant difference in the adequacy of the fragment depending on tumor location (left/right lobe) $(p=0.065)$. 
Table III. Description of cases in which multiple biopsies were performed.

\begin{tabular}{|c|c|c|}
\hline & Number & Comment \\
\hline \multirow[t]{2}{*}{ Primary tumor biopsy } & $12 / 16$ & $\begin{array}{l}\text { In } 75 \%(12 / 16) \text { of cases of metastatic liver lesions, the primary tumor was biopsied and there } \\
\text { was a } 100 \% \text { concurrency between the results. }\end{array}$ \\
\hline & $4 / 16$ & Four patients had late relapse and the primary tumor had already been surgically removed \\
\hline \multirow[t]{2}{*}{ Secondary site biopsy } & $3 / 16$ & $\begin{array}{l}\text { In } 3 \text { cases of metastatic liver lesions, biopsies from suspect adenopathies }(2 / 3) \text { and malignant } \\
\text { portal vein thrombosis }(1 / 3) \text { were also performed and there was a } 100 \% \text { compatibility between } \\
\text { the results. }\end{array}$ \\
\hline & $2 / 11$ & $\begin{array}{l}\text { In } 2 \text { cases of primitive liver tumors, biopsies from suspect adenopathies were also performed. } \\
\text { In } 1 \text { case the specimen obtained wasn't adequate for diagnosis. }\end{array}$ \\
\hline Cytology & $3 / 30$ & $\begin{array}{l}2 / 3 \text { cases of cytology didn't reveal malignant cells; } 1 / 3 \text { cases revealed malignant cells but could } \\
\text { not produce a precise diagnosis }\end{array}$ \\
\hline
\end{tabular}

The diameter of the biopsied lesions ranged from $8 \mathrm{~mm}$ to $95 \mathrm{~mm}$. Starting from the median value of $38 \mathrm{~mm}$, we performed a Chi-squared test to search if there was a statistically significant difference in the adequacy of the fragment regarding tumor size $(p=0.309)$. We did not find that the adequacy of the fragment to be depended on the tumor size.

The median number of needle passages was 1.9 (Range=1-3).

We encountered no severe complications. One patient reported mild pain in the right hypochondria following the procedure, which receded after administration of metamizole.

\section{Discussion}

Liver biopsy continues to be irreplaceable for the diagnosis and staging of the majority of liver tumors. Although percutaneous liver biopsy is a well-established procedure and is often considered the method of choice (7), we believe EUS- liver biopsy (LB) has its own place and should, in many cases, be preferred (8).

Other recent studies using this procedure with similar cohorts managed to acquire proper specimens for histological diagnosis with likelihoods ranging from $88 \%$ to $98 \%$ (9-11). Our study achieved a diagnostic accuracy rate of $97 \%$. There was only one case in which the biopsy provided inadequate material for completely excluding malignancy. This patient had a history of urinary bladder cancer and was diagnosed with multiple liver lesions. The same lesion was also biopsied percutaneously, and the result was: i) moderate fibrosis, ii) grade 3 Ishak, and iii) no signs of malignancy. Thus, in that case, the biopsy that showed no signs of malignancy may have actually been accurate. The two benign biopsies were taken from nonspecific, hypoechoic, hepatic liver lesions in these patients, each one with a history of malignant tumors (mammary carcinoma and NET pancreatic tumor). In both cases, the lesions were in areas of steatosis with signs of regeneration and balonization.
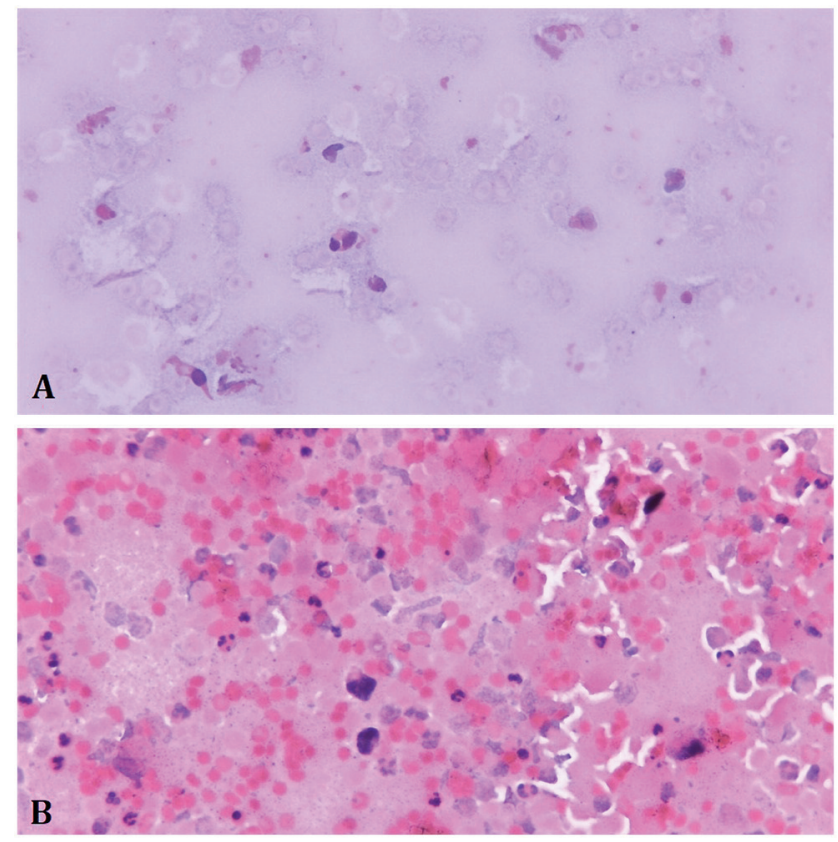

Figure 4. EUS-FNA from malignant liver tumors. A) Smear with rare cells without atypia (Hematoxylin-eosin staining). B) Smear with large, atypical cells (hematoxylin-eosin staining). EUS-FNA: Endoscopic ultrasound- fine needle aspiration. Magnification: $\times 40$.

In $75 \%(12 / 16)$ of patients with liver metastasis, we also biopsied the primary tumor via various methods, such as EUS guidance, percutaneous aspiration, colonoscopy. The results of the liver EUS-guided biopsy and the primary tumor biopsy were consistent. Being able to take biopsy specimens from both the primary tumor and the suspected metastasis can be helpful especially in patients with more than one cancer site or in patients with a primary tumor that can be biopsied via EUS guidance, such as nonspecific liver lesions that need histological analysis (12). At the same time, in 4/16 $(25 \%)$ patients with metastatic lesions we could not perform 
a biopsy from the primary tumor. In these cases, the patients had late relapse and the primary tumor had already been surgically removed. Considering that a biopsy from the liver lesions was the only method to confirm the relapse and the percutaneous route was inaccessible, using EUS guidance had a significant impact on the case management. In 3 patients with liver metastasis, biopsies from suspected metastatic lymph nodes (2/3) and malignant portal vein thrombosis $(1 / 3)$ were also performed in the same procedure. There was a $100 \%$ agreement between the results from the primary tumor and metastatic sites, which served our goal to increase the chance of achieving accurate diagnosis.

In addition to histological analysis, we performed a cytological evaluation in 3 cases, out of which only one revealed malignant cells, without offering a precise diagnosis. On-site pathological evaluation is generally considered to be beneficial with regard to increasing the diagnostic yield of EUS-guided biopsies $(13,14)$. However, given the high diagnostic rates achieved in studies where this evaluation was not available $(11,15,16)$, we considered that it does not justify the extra cost. Furthermore, cytology will only indicate if the specimen is benign/malignant, and offers no guarantee that it will be adequate for histology or immunohistochemistry.

We used a $22 \mathrm{G}$ FNA needle, which is the preferred dimension in other studies as well $(17,18)$, with which we acquired core material in $81 \%$ of cases and fragmented material, used as a cell block, in $19 \%$ of cases. A fine needle biopsy (FNB) specimen differs from an FNA specimen in that it contains core tissue with better preservation of cellular architecture and more likely to provide a higher diagnostic accuracy. Although there are needles that are marketed as "core," the choice of needle does not exclusively determine the definition of "core" (19). When choosing a needle, whether it is $19 \mathrm{G}, 22 \mathrm{G}$ or $25 \mathrm{G}$, one should consider the quality of the biopsy material that can be obtained, the flexibility of the needle and the risk of complications. Thicker needles are more rigid but can provide better samples, while 25G needles are more maneuverable but provide smaller size samples. In our experience, using the $22 \mathrm{G}$ needle can provide with a high-quality specimen, and compact material sufficient for pathological analysis, including immunohistochemistry. To our knowledge, there is no clear recommendation for choosing a specific needle for FNA and the choice falls on the specialist performing the procedure $(20,21)$. With regard to passes, although there is no true consensus, it is thought that 3-4 should suffice, while more of them are likely to yield blood clots (19). Endosonography should be able to assess independently whether the sample is of adequate quality and quantity or additional passes are required (19).

We did not find that the accuracy of the biopsy was depended on the tumor size. Very small tumors can be harder to target, but larger tumors tend to have a necrotic center, which should be avoided. In the latter situation, contrast enhanced ultrasonography can be helpful $(22,23)$.

In this study, the single failed biopsy was taken from the right lobe, but the number of patients was too small to draw any conclusions from. Evaluation and puncture of the right posterior segments (S6/S7) is challenging because they are far from the stomach/duodenum and the maximum length the needle can reach is approximately $6 \mathrm{~cm}$ (24). While patients with metastatic disease usually have at least one lesion that is accessible, those with solitary tumors situated in the right posterior part of the right lobe can be excluded from EUSguided biopsy based on prior imaging. We also agree with the literature that there is no significant difference between the accuracy of the duodenal approach for the accessible part of the right lobe and that of the gastric approach for the left lobe $(10,11,25)$.

EUS-guided liver biopsy is generally a safe procedure with few adverse events. The most common complications such as puncture site bleeding, abdominal pain, and fever, rarely need further therapeutic intervention (26). Until recently, only a small number of EUS-FNA-related needle-tract seeding cases have been reported $(27,28)$, with only one case related to liver EUS-FNA (29). A meta-analysis evaluating the needle track seeding following biopsy of liver lesions has reported a $2.7 \%$ likelihood after percutaneous liver tumor biopsy (30). Although this is thought to be rare, there are no concrete data on the actual frequency of EUS-FNB-related needle-tract seeding, and given the severity of this complication, endosonographers should also be considered, when possible, for shortening the needle path, limiting the number of passes, and surveying the puncture sites.

There are certain factors and actions that may help good quality tissue acquisition and reduce complications. Subtle lesions can be found with repeated back-and-forth scans of the liver by maneuvering the EUS probe and recognizing a distorted pattern of the normal liver parenchyma, vessels, or bile ducts. When performing FNA-FNB, it is helpful to target lesions close to the EUS probe to minimize the distance that is passed through the liver parenchyma. Targeting subcapsular lesions with no liver in front of them should be avoided, given the increased risk of hemorrhage. The central part of the tumor should also be avoided because it may be necrotic (31). While suction increases the quantity of the sample, it makes the specimen bloodier and diminishes the quality of the aspirate, thus, there is no consensus concerning whether it should be used or not (32). The use of a stylet does not seem to improve the diagnostic yield for malignancy and is often found burdensome $(19,33)$.

There are several advantages of using the EUS-LB technique over other methods for acquiring tissue samples. First, EUS-LB provides the endosonographer with a realtime, detailed view of the biopsy needle through the course 
of the lesion, regardless of patient habitus. Therefore, the trajectory can be easily modified in real time with the ability to obtain access to deep, smaller lesions. Second, EUS can also detect lesions $(<1 \mathrm{~cm})$ that are hard to differentiate through trans-abdominal ultrasound or computed tomography $(34,35)$. Third, EUS-LB is usually performed under deep sedation, resulting in a more pleasant experience for the patient and, unlike the percutaneous approach, there is no risk of lesion shifting caused by patient noncompliance. Fourth, EUS-LB is less invasive and associated with a very low complication rate (36). Finally, EUS-LB offers the flexibility to simultaneously obtain biopsy samples from more than one site suspected of malignancy. This can be especially helpful in patients with second or synchronous cancers with multiple lesions that are approachable by EUS-FNA.

EUS-guided biopsy has some disadvantages as well. It is more expensive than percutaneous biopsy due to the added costs of needles, anesthesia and the need for a well-equipped endoscopy laboratory. It is also technically more difficult than percutaneous biopsy and the learning curve is longer $(37,38)$. Calcifications, aerobilia, large gallstones or dense fibrotic scarring bands may shroud the parenchyma and limit the evaluation of the liver. Pre-procedural recognition of these obstacles will help avoid a difficult or failed EUS examination (39). The fragment of tissue obtained is thinner but longer than that obtained with the percutaneous $18 \mathrm{G}$ needles, and it usually meets the criteria proposed by the international societies (40).

This study has some limitations. The number of patients was small, and the design was a single-arm observational study that did not compare the outcomes of EUS-FNA with those of percutaneous US/CT guided biopsy. Therefore, further comparative studies with a large number of patients are necessary to evaluate the advantages/disadvantages of EUS-FNA for liver lesions and better define the indications of this procedure.

In conclusion, EUS-guided biopsy of liver tumors has a high success rate in the procurement of good quality histological fragments, combined with a low rate of complications and decreased patient discomfort. This procedure should be considered especially for liver lesions that are difficult to access via the percutaneous route or when concurrent biopsies are required for accurate diagnosis.

\section{Conflicts of Interest}

The Authors declare no conflicts of interest.

\section{Authors' Contributions}

Conceptualization, VAI and RIC; methodology, VAI; software, VAI; validation, RIC and PAM; formal analysis, VAI; investigation, GAN and $\mathrm{AC}$; resources, GAN and $\mathrm{AC}$; data curation, VAI; writing- original draft preparation, VAI; writing - review and editing, RIC and PAM; visualization, VAI and RIC; supervision, PAM; project administration, VAI and RIC. All Authors have read and agreed to the published version of the manuscript.

\section{References}

1 Dumonceau JM, Deprez PH, Jenssen C, Iglesias-Garcia J, Larghi A, Vanbiervliet G, Aithal GP, Arcidiacono PG, Bastos P, Carrara S, Czakó L, Fernández-Esparrach G, Fockens P, Ginès À, Havre RF, Hassan C, Vilmann P, van Hooft JE and Polkowski M: Indications, results, and clinical impact of endoscopic ultrasound (EUS)-guided sampling in gastroenterology: European Society of Gastrointestinal Endoscopy (ESGE) Clinical Guideline Updated January 2017. Endoscopy 49(7): 695-714, 2017. PMID: 28511234. DOI: $10.1055 / \mathrm{s}-0043-109021$

2 Shioga T, Kondo R, Ogasawara S, Akiba J, Mizuochi S, Kusano H, Mihara Y, Tanigawa M, Kinjyo Y, Naito Y, Kuromatsu R, Nakashima $\mathrm{O}$ and Yano H: Usefulness of tumor tissue biopsy for predicting the biological behavior of hepatocellular carcinoma. Anticancer Res 40(7): 4105-4113, 2020. PMID: 32620659. DOI: 10.21873/anticanres.14409

3 Sterlacci W, Sioulas AD, Veits L, Gönüllü P, Schachschal G, Groth S, Anders M, Kontos CK, Topalidis T, Hinsch A, Vieth M, Rösch T and Denzer UW: 22-gauge core vs. 22-gauge aspiration needle for endoscopic ultrasound-guided sampling of abdominal masses. World J Gastroenterol 22(39): 8820-8830, 2016. PMID: 27818598. DOI: $10.3748 /$ wjg.v22.i39.8820

4 Hasan MK, Kadkhodayan K, Idrisov E, Ali S, Rafiq E, Ben-Ami Shor D, Abdel-Jalil A, Navaneethan U, Bang J, Varadarajulu S, Hawes R and Pernicone P: Endoscopic ultrasound-guided liver biopsy using a 22-G fine needle biopsy needle: a prospective study. Endoscopy 51(9): 818-824, 2019. PMID: 31365947. DOI: 10.1055/a-0967-3640

5 Eskandari A, Koo P, Bang H, Gui D and Urayama S: Comparison of endoscopic ultrasound biopsy needles for endoscopic ultrasound-guided liver biopsy. Clin Endosc 52(4): 347-352, 2019. PMID: 31288504. DOI: 10.5946/ce.2019.005

6 Veitch AM, Vanbiervliet G, Gershlick AH, Boustiere C, Baglin TP, Smith LA, Radaelli F, Knight E, Gralnek IM, Hassan C and Dumonceau JM: Endoscopy in patients on antiplatelet or anticoagulant therapy, including direct oral anticoagulants: British Society of Gastroenterology (BSG) and European Society of Gastrointestinal Endoscopy (ESGE) guidelines. Endoscopy 48(4): 385-402, 2016. PMID: 26890676. DOI: 10.1055/s-0042-102652

7 Tian G, Kong D, Jiang T and Li L: Complications after percutaneous ultrasound-guided liver biopsy: a systematic review and meta-analysis of a population of more than 12,000 patients from 51 cohort studies. J Ultrasound Med 39(7): 13551365, 2020. PMID: 31999005. DOI: 10.1002/jum.15229

8 Liu LI, Ahn E, Studeman K, Campbell K and Lai J: Primary hepatic carcinosarcoma composed of hepatocellular carcinoma, cholangiocarcinoma, osteosarcoma and rhabdomyosarcoma with poor prognosis. Anticancer Res 40(4): 2225-2229, 2020. PMID: 32234918. DOI: 10.21873/anticanres.14184

9 Akay E, Atasoy D, Altınkaya E, Koç A, Ertan T, Karaman H and Caglar E: Endoscopic ultrasound-guided fine needle aspiration using a 22-G needle for hepatic lesions: Single-center experience. Clin Endosc 54(3): 404-412, 2021. PMID: 33291191. DOI: $10.5946 / \mathrm{ce} .2020 .065$ 
10 Chen F, Bao H, Deng Z, Zhao Q, Tian G and Jiang TA: Endoscopic ultrasound-guided sampling using core biopsy needle for diagnosis of left-lobe hepatocellular carcinoma in patients with underlying cirrhosis. J Cancer Res Ther 16(5): 1100-1105, 2020. PMID: 33004754. DOI: 10.4103/jert. JCRT_723_19

11 Ichim VA, Chira RI, Mircea PA, Nagy GA, Crisan D and Socaciu MA: Accuracy of endoscopic ultrasound-guided biopsy of focal liver lesions. Med Ultrason 22(1): 20-25, 2020. PMID: 32096783. DOI: $10.11152 / \mathrm{mu}-2078$

12 Raptakis T, Boura P, Tsimpoukis S, Gkiozos I and Syrigos KN: Endoscopic and endobronchial ultrasound-guided needle aspiration in the mediastinal staging of non-small cell lung cancer. Anticancer Res 33(6): 2369-2376, 2013. PMID: 23749884

13 Polkowski M, Larghi A, Weynand B, Boustière C, Giovannini M, Pujol B, Dumonceau JM and European Society of Gastrointestinal Endoscopy (ESGE): Learning, techniques, and complications of endoscopic ultrasound (EUS)-guided sampling in gastroenterology: European Society of Gastrointestinal Endoscopy (ESGE) Technical Guideline. Endoscopy 44(2): 190206, 2012. PMID: 22180307. DOI: 10.1055/s-0031-1291543

14 Bhatia V and Varadarajulu S: Endoscopic ultrasonographyguided tissue acquisition: How to achieve excellence. Dig Endosc 29(4): 417-430, 2017. PMID: 28140485. DOI: 10.1111/ den. 12823

15 Lee YN, Moon JH, Kim HK, Choi HJ, Choi MH, Kim DC, Lee TH, Lee TH, Cha SW, Kim SG and Kim YS: Usefulness of endoscopic ultrasound-guided sampling using core biopsy needle as a percutaneous biopsy rescue for diagnosis of solid liver mass: Combined histological-cytological analysis. J Gastroenterol Hepatol 30(7): 1161-1166, 2015. PMID: 25684303. DOI: 10.1111 /jgh.12922

16 Chira RI, Chira A, Ichim VA, Nagy GA, Florea A, Crisan D and Popovici B: Endoscopic ultrasound-guided fine-needle aspiration (EUS-FNA) of paraesophageal lung tumors - diagnostic yield and added value. Med Ultrason 21(4): 377-381, 2019. PMID: 31765444. DOI: $10.11152 / \mathrm{mu}-2184$

17 Ichim VA, Chira RI and Mircea PA: Diagnostic yield of endoscopic ultrasound-guided biopsy of focal liver lesions. Med Pharm Rep 92(1): 15-20, 2019. PMID: 30957081. DOI: 10.15386/cjmed-1066

18 Sbeit W, Kadah A, Mahamid M, Pellicano R, Mari A and Khoury T: A state-of-the-art review on the evolving utility of endoscopic ultrasound in liver diseases diagnosis. Diagnostics (Basel) 10(8): 512, 2020. PMID: 32717886. DOI: $10.3390 /$ diagnostics 10080512

19 Varadarajulu S, Hasan MK, Bang JY, Hebert-Magee S and Hawes RH: Endoscopic ultrasound-guided tissue acquisition. Dig Endosc 26 Suppl 1: 62-69, 2014. PMID: 24033879. DOI: 10.1111/den.12146

20 Vozzo C, Saleh MA, Drake R and Chahal P: Endoscopic ultrasound-guided liver biopsy: needle types and suction methods. VideoGIE 6(11): 485-486, 2021. PMID: 34765836. DOI: $10.1016 /$ j.vgie.2021.06.007

21 Siddique DA, Rahal MA, Trevino K, Wu HH and Al-Haddad MA: Endoscopic ultrasound-guided sampling of solid pancreatic lesions: a comparative analysis of 25 gauge versus 22 gauge core biopsy needles. Anticancer Res 40(10): 5845-5851, 2020. PMID: 32988914. DOI: 10.21873 /anticanres.14603
22 Seicean A, Badea R, Moldovan-Pop A, Vultur S, Botan EC, Zaharie T, Săftoiu A, Mocan T, Iancu C, Graur F, Sparchez Z and Seicean R: Harmonic contrast-enhanced endoscopic ultrasonography for the guidance of fine-needle aspiration in solid pancreatic masses. Ultraschall Med 38(2): 174-182, 2017. PMID: 26274382. DOI: 10.1055/s-0035-1553496

23 Oh D, Seo DW, Hong SM, Jun JH, Song TJ, Park DH, Son BK, Lee SS, Lee SK and Kim MH: The usefulness of contrastenhanced harmonic EUS-guided fine-needle aspiration for evaluation of hepatic lesions (with video). Gastrointest Endosc 88(3): 495-501, 2018. PMID: 29859228. DOI: 10.1016/j.gie. 2018.05.019

24 Tsujino T, Samarasena JB and Chang KJ: EUS anatomy of the liver segments. Endosc Ultrasound 7(4): 246-251, 2018. PMID: 30117487. DOI: 10.4103/eus.eus_34_18

25 Oh D, Seo DW, Hong SM, Song TJ, Park DH, Lee SS, Lee SK and Kim MH: Endoscopic ultrasound-guided fine-needle aspiration can target right liver mass. Endosc Ultrasound 6(2): 109-115, 2017. PMID: 28440236. DOI: 10.4103/23039027.204813

26 Mohan BP, Shakhatreh M, Garg R, Ponnada S and Adler DG: Efficacy and safety of EUS-guided liver biopsy: a systematic review and meta-analysis. Gastrointest Endosc 89(2): 238246.e3, 2019. PMID: 30389469. DOI: 10.1016/j.gie.2018.10.018

27 Mizuide M, Ryozawa S, Fujita A, Ogawa T, Katsuda H, Suzuki M, Noguchi T and Tanisaka Y: Complications of endoscopic ultrasound-guided fine needle aspiration: a narrative review. Diagnostics (Basel) 10(11): 964, 2020. PMID: 33213103. DOI: 10.3390/diagnostics10110964

28 Jenssen C, Alvarez-Sánchez MV, Napoléon B and Faiss S: Diagnostic endoscopic ultrasonography: assessment of safety and prevention of complications. World J Gastroenterol 18(34): 46594676, 2012. PMID: 23002335. DOI: 10.3748/wjg.v18.i34.4659

29 Kasi M, Rashid S, Wallace SAJ, Sujendran V, Griffiths B, Butler A, Gibbs P, Sreedharan L, Zaitoun AM, Venkatachalapathy S, James MW and Aithal GP: Seeding of hepatocellular carcinoma into the stomach wall following endoscopic ultrasound and fineneedle aspiration biopsy. Oxf Med Case Reports 2018(7): omy039, 2018. PMID: 30046450. DOI: 10.1093/omcr/omy039

30 Silva MA, Hegab B, Hyde C, Guo B, Buckels JA and Mirza DF: Needle track seeding following biopsy of liver lesions in the diagnosis of hepatocellular cancer: a systematic review and meta-analysis. Gut 57(11): 1592-1596, 2008. PMID: 18669577. DOI: $10.1136 /$ gut.2008.149062

31 Yousaf MN, Cai G and Aslanian HR: EUS evaluation of liver lesions. VideoGIE 3(1): 31-33, 2017. PMID: 29905169. DOI: 10.1016/j.vgie.2017.09.012

32 Wallace MB, Kennedy T, Durkalski V, Eloubeidi MA, Etamad R, Matsuda K, Lewin D, Van Velse A, Hennesey W, Hawes RH and Hoffman BJ: Randomized controlled trial of EUS-guided fine needle aspiration techniques for the detection of malignant lymphadenopathy. Gastrointest Endosc 54(4): 441-447, 2001. PMID: 11577304. DOI: 10.1067/mge.2001.117764

33 Wani S, Gupta N, Gaddam S, Singh V, Ulusarac O, Romanas M, Bansal A, Sharma P, Olyaee MS and Rastogi A: A comparative study of endoscopic ultrasound guided fine needle aspiration with and without a stylet. Dig Dis Sci 56(8): 2409-2414, 2011. PMID: 21327919. DOI: 10.1007/s10620-011-1608-Z

34 Singh P, Mukhopadhyay P, Bhatt B, Patel T, Kiss A, Gupta R, Bhat $\mathrm{S}$ and Erickson RA: Endoscopic ultrasound versus $\mathrm{CT}$ scan 
for detection of the metastases to the liver: results of a prospective comparative study. J Clin Gastroenterol 43(4): 367373, 2009. PMID: 18981929. DOI: 10.1097/MCG. $0 \mathrm{~b} 013 \mathrm{e} 318167 \mathrm{~b} 8 \mathrm{cc}$

35 Hafez HA, Okasha HH, Hashem AM, Hassany M and El-Nahaas SM: The Role of EUS and EUS-FNA in detection of small sized liver metastatic lesions in patients with pancreatic and gastrointestinal primary malignancy. The Medical Journal of Cairo University 88(6): 1219-1225, 2020. DOI: $10.21608 / \mathrm{mjcu}$. 2020.110872

36 Sbeit W, Kadah A, Mari A, Mahamid M and Khoury T: A comprehensive narrative review on the evolving role of endoscopic ultrasound in focal solid liver lesions diagnosis and management. Diagnostics (Basel) 10(9): 688, 2020. PMID: 32932960. DOI: 10.3390/diagnostics 10090688

37 Mertz H and Gautam S: The learning curve for EUS-guided FNA of pancreatic cancer. Gastrointest Endosc 59(1): 33-37, 2004. PMID: 14722544. DOI: 10.1016/s0016-5107(03)02028-5
38 Eloubeidi MA and Tamhane A: EUS-guided FNA of solid pancreatic masses: a learning curve with 300 consecutive procedures. Gastrointest Endosc 61(6): 700-708, 2005. PMID: 15855975. DOI: 10.1016/s0016-5107(05)00363-9

39 Bhatia V, Hijioka S, Hara K, Mizuno N, Imaoka H and Yamao $\mathrm{K}$ : Endoscopic ultrasound description of liver segmentation and anatomy. Dig Endosc 26(3): 482-490, 2014. PMID: 24355092. DOI: $10.1111 /$ den.12216

40 Johnson KD, Laoveeravat P, Yee EU, Perisetti A, Thandassery $\mathrm{RB}$ and Tharian B: Endoscopic ultrasound guided liver biopsy: Recent evidence. World J Gastrointest Endosc 12(3): 83-97, 2020. PMID: 32218888. DOI: 10.4253/wjge.v12.i3.83

Received October 22, 2021

Revised December 9, 2021 Accepted December 21, 2021 